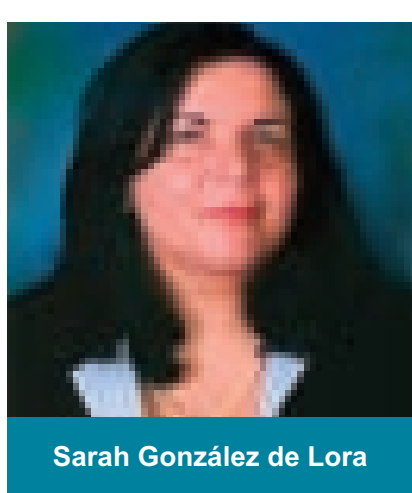

Uno de los propósitos es gestionar y proponer a la Rectoría, en colaboración con las Vicer Académica y de Postgrado, as relaciones interinstitucionales, la creación de alianzas estratégicas y la firma de con requieran en las diferentes instancias para fortalecer las ofertas de la PUCMM en las áreas de docencia, investigación y servicio.

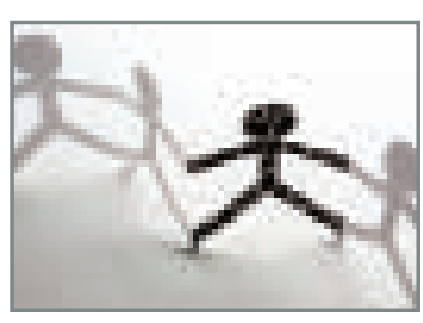

\section{Entrevista a Sarah González de Lora, Vicerrectora de Relaciones Interinstitu- cionales, de Investigación e Innovación de la PUCMM}

Sarah González de Lora ha estado vinculada a la Pontificia Universida Católica Madre y Maestra (PUCMM) desde 1978. Ha desempeñado los cargos de Investigadora y Encargada de Centro Latinoamericano de Investigación y Desarrollo en Educación Matemática (CLIDEM), auspiciado por International Development and $\mathrm{Re}$ search Centre (IDRC) y Canadian International Development Agency (CIDA) en el período 1982-1992. Fue la Coordinadora General del Proyecto de Capacitación de Maestros del Nive Básico (PRODEP) en la PUCMM, financiado por el Banco Mundial. Además, ejerció los cargos de Directora del Departamento de Educación Decana de la Facultad de Ciencias Sociales y Humanidades desde 1997 hasta 2004 y de Vicerrectora Académica en el período 2004-2006. Desde el 12 de julio de 2006 está al frente de esta nueva Vicerrectoría, por cuya labor la estamos entrevistando para este número del Cuaderno de Pedagogía Universitaria.

1. ¿Cuáles son los propósitos generales de esta Vicerrectoría?

Trabajamos conjuntamente en Santiago y en Santo Domingo, en las tres dimensiones que le dan nombre a esta Unidad. Uno de los propósitos es gestionar y proponer a la Rectoría, en colaboración con las Vicerrectorías Académica y de Postgrado, las relaciones interinstitucionales, la creación de alianzas estratégicas y la firma de diferentes instancias para fortalecer las ofertas de la PUCMM en las áreas de docencia, investigación y servicio Existen diversos tipos de relaciones, como los convenios con otras universidades para ofrecer estudios de postgrado, o por ejemplo, el Convenio Magallanes, que propicia la movilidad de estudiantes entre Europa y América vez aprobados por la Rectoría se elaboran en esta Vicerrectoría y luego se delegan a la unidad correspondiente para su ejecución

Una de las relaciones interinstitucionales que estamos priorizando es con el Centro Internacional para la Innovación en Tecnología y Adminis tración (CITAM). Este organismo planea ofrecer programas de maestría y educación permanente para los países de la región cercana de América Latina, con la titulación de Rocheste Institute Technology (RIT) de Estados convenios que se requieran en las Latina. Los acuerdos como éstos, una
Unidos y de la PUCMM. Hemos conseguido que el CITAM se vincule con e gobierno para dar respuesta a necesidades tecnológicas y de gerencia en el marco de la globalización. El Centro de Exportación e Inversión de la República Dominicana (CEI-RD) financió traer exertos del RIT para realizar un diagnostico y presentar estrategias para hace más competitivas las zonas francas del país. Con este fin, se consiguieron sesenta (60) medias becas de la Secretaría de Estado de Educación Superior, Ciencia y Tecnología (SEESCyT) para cuatro programas de maestrías del RIT en las áreas de administración de manufacturas, en redes, en telecomunicaciones en administración de servicios. Elconvenio para estas becas fue firmado po nuestra Universidad y la SEESCyT, el viernes 11 de mayo del presente año.

tro propósito de la Vicerrectoría es servir a las necesidades de investigación existentes y futuras de la PUCMM. Esto se llevará a cabo ofreciendo apoyo a las personas o grupos interesados, construyendo las infraestructuras necesarias dentro del contexto de la investigación y la innovación para el desarrollo, fundamentándose en altos estándares éticos y sirviendo de canal para la transferencia del conocimiento, así como para el manejo de la propiedad intelectual.

Dependiendo del proyecto, las dimensiones de relaciones interinstitucionales, investigación e innovación pueden aparecer integradas. Las relaciones con las instituciones que se acercan a la PUCMM, o viceversa, sea de educación superior o no, se gestio- nan a través de esta instancia. Por ejemplo, la Empresa Distribuidora de Electricidad del Norte (Edenorte), solicitó al Centro de Estudios Urbanos y Regionales (CEUR), que es uno de los Centros que dependen de esta Vicerrectoría, la realización de un estudio sobre las mejores vías para acercars a las comunidades, en vistas de mejorar la comunicación con los usuarios del servicio eléctrico en varias dimensiones. En el país, este tema ha estado marcado por una trayectoria de desconfianza hacia la empresa de electricidad. EI CEUR está investigando sobre las estrategias de abordaje socia más convenientes. El trabajo está teniendo éxito, pues el componente de la Universidad aporta credibilidad en las comunidades. Algunas de ellas, que antes no pagaban los servicios consumidos ahora están realizando sus pagos. Este es el caso de un proyecto en el cual investigación y desarrollo están integrados

2. Una de las acciones ejecutadas por esta Vicerrectoría cuando fue creada era elaborar un plan a cuatro años para la promoción y la ejecución de investigaciones e innovaciones en la PUCMM, en varias etapas. ¿Cuáles acciones de este plan se han cumplido y qué se está haciendo actualmente para llevar a cabo los objetivos planteados?

Para este plan, la Universidad ha retomado el camino ya recorrido en relación a la investigación, con la cual ha tratado de mantenerse siempre vinculada. A pesar de las dificultades que
Otro propósito de la Viceectoría es servir a las necesidades de investigaciones existentes y futuras de la PUCMM.

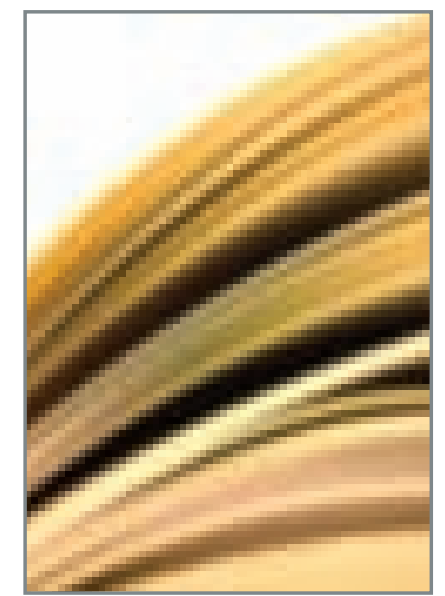

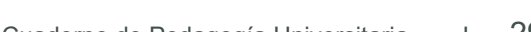




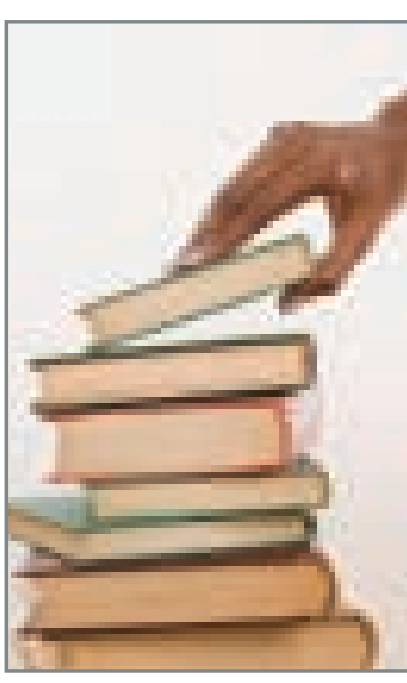

A pesar de las dificultades que las instituciones de los países en desarrollo enfrentan para sustentar estructuras de investigación la PUCMM ha tratado de mantenervi de la investigación. En 1974 se creó el Centro de Investigaciones, el cual trabajo por más de una década con lineamientos parecidos a los de esta Vicerrectoría en el área de investigación.

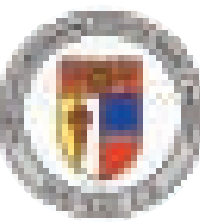

las instituciones de los países en desarrollo enfrentan para sustentar estigación, la PUCMM ha tratado de mantener viva la dimensión de la investigación. En 1974 se creó el Centro de Investigaciones, el cual trabajó por más de una década con lineamie esta Vicerrectoría en el área de investigación. Luego de la fuerte crisis macroeconómica de los años 80 , designada como "década pérdida" en América Latina, quedaron algunos proyectos que la Universidad pudo seguir apoyando. EI CEUR y los actuales proyectos de enseñanza de la lengua española y de la matemática (CETT, TEF y CEIE) ${ }^{1}$ son algunas de las herencias de esa época.

Dentro de las acciones ejecutadas está la conformación de un Consejo de Investigación, integrado por los directores de los diferentes centros proyectos asi como decanos y/o representantes de las facultades de nuestra Universidad. Las fases de ejecución del plan se han concebido de la siguiente manera:

En la primera fase, se creó la estructura de gestión, que es esta Vicerrectoría. Estamos trabajando en la normativa que regulará la investigación en la PUCMM y en el establecimiento de prioridades temáticas a corto plazo las cuales abarcan economía, educación, salud, ambiente, energías alternativas, valores, emprendimiento,

politica y tecnologia. Otras acciones que se están definiendo son la vinculación de la comunidad universitaria los centros de investigación existentes, $y$ los esquemas de financiamiento de investigación e innovación. También estamos dando importancia a la capacitación para elaborar propuestas a organismos internacionales y a las estrategias de obtención de información y recursos. En el caso de la Vicerectoría de Postgrado, al ser también una Unidad de reciente creación, contemplamos trabajar en coordinación con ella, a partir de las políticas de investigación en las que ya han estado trabajando.

En la segunda fase, se priorizó creación de una unidad de Gestión de la Investigación. Buscamos fortalecer la investigación vinculada al currículo, po lo cual durante el mes de junio nos reuniremos con las Facultades y los Departamentos para hacer un análisis de las Fortalezas, Oportunidades, debilidades y Amenazas (FODA) con las unidades vinculadas a la investigación. De manera particular, se han acercado a esta Vicerrectoría directores de carrera, a los cuales hemos comenzado apoyar en las necesidades específicas de su Departamento relacionadas con la investigación.

Otra de las metas es el fortalecimiento de los centros de investigación existentes. Queremos determinar cuáles son las mejores estrategias para su 1 En español, la traducción de estas siglas es: Centro de Excelencia para la Capacitación de Maestros,
Aprendizaje Efectivo de la Matemática y Consorcio de Evaluación e Investigación Educativa, respectiAprendizaje
vamente. sostenibilidad de manera que tengan na permanencia garantizada, as como la socialización permanente de sus labores. Buscamos, además, crear fondos concursables para investigación en las áreas prioritarias, aportando "fondos semilla" para la creación de núcleos de investigación de especialistas, promoviendo las publicaciones y ofreciendo servicios de formación y apoyo metodológico.

En la tercera fase nos proponemos e desarrollo de los programas y estrategias de investigación. Una vez recogidos los diagnósticos de las fases 1 y 2 , se elaborará un programa de investigación que se llevaría a cabo en los tres años siguientes. El mismo incluirá un plan de trabajo, el presupuesto, el plan de monitoreo y la evaluación continua de los programas que se ejecuten. El consenso sobre las líneas de investigación de interés para la Universidad es vital para esta fase.

3. ¿Qué políticas de difusión está llevando a cabo esta Vicerrectoría para dar a conocer lo que hace?

Se están diseñando brochures y páginas electrónicas de la Vicerrectoría y de los centros de investigación para ponerlas en el portal electrónico de la Universidad, pues hay mucha información que no llega a todos los sectores interesados.

Tenemos pautadas reuniones en las facultades y los centros en junio de 2007 y la realización de un foro en ese mismo mes para difundir el trabajo ac- tual de los centros y departamentos. Estos foros serán organizados con periodicidad, pues sabemos que muchos de los integrantes de la comunidad universitaria desconocen el trabajo que se desarrolla en cada uno de ellos. Hemo estado utilizando el correo electrónico de la Universidad para anunciar las convocatorias a fondos de investigación tales como las del Fondo Brugal para la Educación y la Investigación, del Fondo para el Fomento de Investigación Económica y Social (FIES), los Fondos para el Desarrollo Institucional de la Sociedad Civil Dominicana (FONDESIN) y el Fondo $\mathrm{Na}$ cional de Innovación y Desarrollo Científico y Tecnológico de la SEESCYT. Queremos llegar directamente al profesorado y a los estudiantes, en los casos que aplique. La publicación de una revista como órgano de difusión se hará imprescindible para el logro de este objetivo.

4. ¿Cómo un profesor de la PUCMM puede presentar a esta Vicerrectoría un proyecto de investigación? ¿Qué requisitos son necesarios?

Todos los profesores pueden presenta una propuesta de investigación. Estamos elaborando un protocolo para ello. Básicamente el profesor debe escribi una propuesta sencilla y presentarla a la Dirección de su Departamento. Si se considera adecuada, se eleva a la $\mathrm{Fa}$ cultad y se presenta al Consejo de Investigación para su aprobación final. Estamos motivando especialmente la participación en los concursos de la tercera fase nos proponemos el desarrollo de os program recogidos los diagnósticos de las fases 1 y 2 , se elaborará un programa de investigación que se llevaría abo en los guientes.

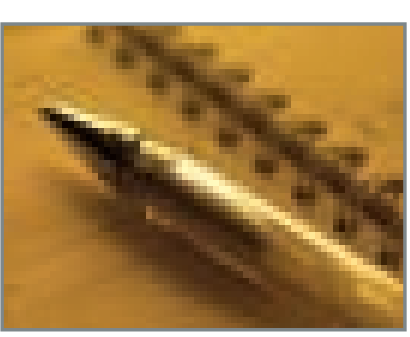

c 31 
investigación para promover proyectos auto-financiables.

En la Facultad de Ingeniería están interesados en la creación de un Instituto de Investigación de Materiales (INMATECA), el cual trabajaría en la cerEn el área de Ingeniería tenemos también a un profesor interesado en la producción de biodiesel a partir de la Higuereta. Esto ya se ha hecho en el país pero hay una variedad $d e$ Brasil, que da sultados. El profesor está presentando el proyecto a Fondo Brugal para la Educación y la Investigación.

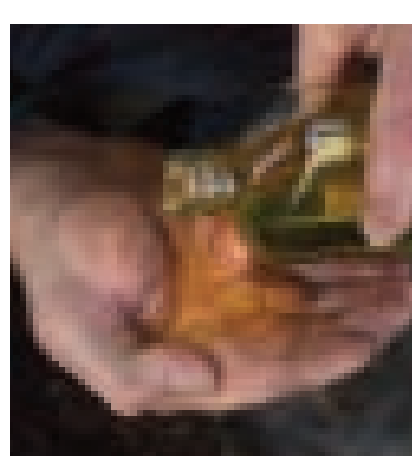

tificación de la calidad de productos. También esta Facultad está involucrada con el área prioritaria de energías alternativas, y en la Faculta de Ciencias Sociales y Administrativas, el Area de Negocios tiene interés en abrir un centro de investigación que abarque diferentes opciones de investigación. Ya han iniciado el Proyecto Global Entrepreneurship Monitor (GEM), el cual es liderado por London Business School de Inglaterra y cuyo objetivo es investigar las prácticas emprendedoras en el país y a nive mundial.

5. ¿Qué tipos de mecanismos de motivación y de formación están planificados para que los docentes se involucren en proyectos de innovación e investigación?

Para elaborar la normativa de investigación estamos consultando lo que se hace en otras universidades. Consideramos que la investigación debe tener una recompensa económica, de derecho de autor, además de la satisfacción profesional y personal que se recibe a participar en investigaciones. Queremos motivar la participación a través de concursos, destinar parte de la carga académica a la investigación, y ofrecer formación y apoyo metodológico. Todo esto se realizará en coordinación con las instancias universitarias pertinentes.

6. ¿Qué se entiende por innovación desde esta Unidad?

Es un término que se está usando mucho dentro del contexto de la globalización. Se asume como la respuesta los cambios continuos que nos presenta nuestro mundo de hoy; cada día enfrentamos nuevos retos a los que sólo podemos dar respuestas mediante innovaciones en los procesos, en los productos y en los servicios. En Estados Unidos pueden considerar una maestría específica como innovación. Por ejemplo, hace pocos días asistimos a la presentación del trabajo final de unos estudiantes de la Carrera de Ingeniería Electromecánica, sobre producción de biodiesel con aceite de cocina quemado, el que sobra después de cocinar. Los estudiantes hicieron funcionar un motor, demostrando todo el proceso.

Aunque todavía estamos en una fase de organización, percibimos que en la Universidad ha habido muy buena acogida a la idea del fortalecimiento de la investigación, pues tenemos muchos profesores con ideas interesantes presentando propuestas. La efervescencia con la investigación refleja la búsqueda de respuestas a tantos retos que tenemos como país. Eso es lo que queremos apoyar, lo cual también implica un gran reto.

\section{Entrevista a Radhamés Mejía, Vice- rrector a cargo del Centro de Investigaciones en Educación y Desarrollo Humano (CIEDHU- MANO), de la PUCMM}

Proveniente del área de la investigación social, Radhamés Mejía inició su trayectoria en la Pontificia Universidad Católica Madre y Maestra (PUCMM) en 1968. Asumió la apertura del Recinto Santo Tomás de Aquino en 1981 después de desempeñar en el Campus de Santiago las posiciones de Directo del Departamento de Ciencias Sociales primer Director del Centro de Investigaciones y Vicerrector Académico desde el 1975 hasta su ida a Santo Domingo. Ahí ejerció como Vicerrector Ejecutivo hasta el año 2004. Es miembro de numerosas organizaciones internacionales relacionadas a Educación Superior, siendo condecorado por el gobierno francés con las Palmas Académicas y por el Papa Juan Pablo II con la Orden de San Silvestre. Recientemente, la Universidad de las Antillas y Guyana le otorgó un Doctorado Honoris Causa. Es el fundador y Director del CIEDHUMANO, por cuya labor investigativa el Cuaderno de Pedagogía Universitaria lo entrevista para este número.

1. ¿Por qué la PUCMM decide formar este centro de investigación? ¿Qué motivaciones la mueven?

Si retomamos la historia de la PUCMM ésta nace con pocas carreras, una de ellas es la Educación. Siguiendo la va- loración que la Iglesia hace de la educación como elemento fundamental de desarrollo nacional, las universidades católicas siempre tienen como uno de sus objetivos contribuir al mejoramiento de los sistemas educativos en los que se encuentran.

El surgimiento del CIEDHUMANO es coherente con esta trayectoria. Está motivado por el convencimiento de que el discurso educativo es mucho más complejo de lo que se piensa. Para poder dar respuesta a los desafíos que éste plantea, se requiere un conocimiento a fondo de los factores involucrados, desde lo que ocurre en el aula, hasta la gestión de todo el sistema y su proyección al futuro. Una indimensiones se hace imprescindible, $y$ sin embargo, muchas de las decisiones se toman con escaso uso de los resultados de estudios hechos con rigor. Lamentablemente, con frecuencia solemos encontrar ausencia de datos bien fundamentados o la ejecución de políticas sin evaluar el contexto previo. En el CIEDHUMANO, por tanto, buscamos enriquecer el debate nacional sobre los problemas educativos aportando resultados de investigaciones hechas con calidad científica, para contribuir así al planteamiento de soluciones pertinentes a nuestra realidad. vestigación permanente de esas

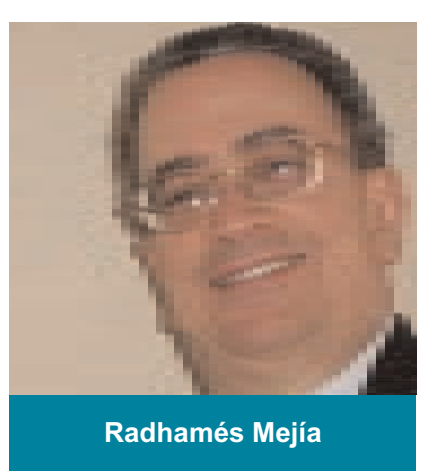

"El conocimiento pedagógico no es algo que se aprende cursando estudios docentes, el sitio donde se aprende a ser maestro es la escuela, el aula". 